\title{
FRACTIONAL DIFFERENTIATION IN THE SELF-AFFINE CASE III. THE DENSITY OF THE CANTOR SET
}

\author{
N. PATZSCHKE AND M. ZÄHLE \\ (Communicated by Charles C. Pugh)
}

\begin{abstract}
We compute the fractional density of the middle-third Cantor measure explicitly. Its numerical value is $0.62344 \ldots$.
\end{abstract}

\section{INTRODUCTION}

A special result of Part I of this paper (see [11]) concerns self-similar random processes $X(t)$, i.e., random real-valued functions on $\mathbf{R}$ that have stationary increments and the scaling property

$$
r^{-\alpha} X(r(\cdot))=X \quad \text { (in distribution) }
$$

for any $r>0$, where $\alpha$ is the scaling exponent. It is shown that the fractional derivatives in the mean

$$
d_{\alpha} X(t):=\lim _{T \rightarrow \infty} \frac{1}{T} \int_{0}^{T} \frac{\left|X\left(t+e^{-u}\right)-X(t)\right|}{e^{-u \alpha}} d u
$$

exist at almost all $t$ with probability 1 and equal a constant under certain ergodicity conditions. In a later part we will prove similar relations for a general class of strictly self-affine functions as considered in analysis (see, e.g., Kamae [7], Kôno [8, 9], Tricot [15, 16], and $\mathrm{Hu}$ and Lau [5, 6]). These functions do not possess the classical fractional derivatives in the sense of Liouville or of Marchaud; however, the fractional Cesáro derivatives mentioned above are meaningful, and their connection with self-affine functions is the same as the connection between classical derivatives and linear functions. A version of the derivatives of order $\frac{1}{2}$ arises in natural manner in Itô calculus with respect to such functions (cf. Bedford and Kamae [2]).

In order to demonstrate some general ideas we will deal with the special case of the Cantor function that was treated in Bedford and Fisher [1] with other tools. Our probabilistic method allows an explicit calculation of the derivative (or of the density of the Cantor set in the language of measures, see Theorem 2). This has been an open problem until now.

Received by the editors May 14, 1991.

1991 Mathematics Subject Classification. Primary 28A75, 58F13, 60D05. 


\section{Fractal properties of the CANTOR Measure}

Define the similarities $S_{1}, S_{2}: \mathbf{R} \rightarrow \mathbf{R}$ by

$$
S_{1}(x)=\frac{x}{3}, \quad S_{2}(x)=\frac{x}{3}+\frac{2}{3} .
$$

The decreasing sequence $C_{n}$ of compact sets given by $C_{0}=[0,1], C_{n}=$ $S_{1} C_{n-1} \cup S_{2} C_{n-1}$ determines the middle-third Cantor set $C=\bigcap_{n=0} C_{n}$, which is self-similar, i.e., $C=S_{1} C \cup S_{2} C$.

It is well known that the Hausdorff dimension $D$ of $C$ equals $\ln 2 / \ln 3$. The normalized $D$-dimensional Hausdorff measure on $\mathbf{R}$ restricted to $C$ is said to be the Cantor measure $\varphi$. It may be constructed as the weak limit of the measures

$$
\varphi_{n}:=\left(\frac{3}{2}\right)^{n} \mathscr{L}\left\llcorner C_{n}\right.
$$

where $\mathscr{L}$ is Lebesgue measure and $L$ means the restriction of a measure to a set. Obviously, we obtain the invariance property

$$
\varphi=\frac{1}{2} \varphi \circ S_{1}^{-1}+\frac{1}{2} \varphi \circ S_{2}^{-1} .
$$

(For more details see Falconer [3].) $\Phi(x):=\varphi([0, x]), x \in[0,1]$, is the Cantor function or the so-called devil's stairway. It satisfies the scaling relation $\Phi(x)=2 \Phi(x / 3)$.

In the next section we will recall a similar scaling property of the tangential measure of $\varphi$ at a point chosen at random according to the (probability) measure $\varphi$.

\section{THE RANDOM TANGENTIAL MEASURE \\ AT THE TYPICAL POINT OF THE CANTOR SET}

Define the distribution $P_{0}$ of a random measure on $\mathbf{R}$ related to $\varphi$ by translating the origin into a point chosen at random with respect to $\varphi$ :

$$
P_{0}=\int 1_{(\cdot)}\left(T_{x} \varphi\right) \varphi(d x)
$$

where $T_{x} \mu$ for a measure $\mu$ on $\mathbf{R}$ denotes the translated measure $\mu((\cdot)+x)$. For $r>0$ let $S_{D, r}$ be the "renormalized" similitudes on the space of locally finite measures on $\mathbf{R}$ given by $S_{D, r} \mu=r^{D} \mu(\cdot / r)$. With their help we define a sequence of distributions of random measures

$$
P_{n}=P_{0} \circ S_{D, 1 / 3^{n}}
$$

Because of the simple structure of the Cantor set, it is not difficult to see that the $P_{n}$ converge weakly in the vague topology on the space of locally finite measures towards a limit distribution $P$ as $n \rightarrow \infty$. Moreover, if the function $f(\mu)$ depends only on $\mu\left\llcorner\left(\left[0,3^{m}\right]\right)\right.$ then we have

$$
\int f(\mu) P_{m+n}(d \mu)=\int f(\mu) P_{m}(d \mu), \quad n \in \mathbf{N} .
$$


(For strong proofs see Ulrich Zähle [17].) Consequently, if $f(\mu)$ depends only on $\mu\llcorner[0,1]$ then we get

$$
\int f(\mu) P(d \mu)=\int f(\mu) P_{0}(d \mu) .
$$

By construction,

$$
P \circ S_{D, 1 / 3}^{-1}=P=P \circ S_{D, 3}^{-1} \text {. }
$$

Remark. Similarly as in Patzschke and Ulrich Zähle [12] (for a more general setting of self-similar sets) one can prove the convergence

$$
\frac{1}{T} \int_{0}^{T} P_{0} \circ S_{D, 1 / t} d t \stackrel{T \rightarrow \infty}{\Longrightarrow} P .
$$

Since $S_{D, 1 / s} \circ S_{D, 1 / t}=S_{D, 1 / s t}$, it follows that $P$ is $D$-scaling, i.e.,

$$
P \circ S_{D, r}^{-1}=P, \quad r>0 .
$$

But for the aims of this paper the weaker invariance (3) is sufficient.

Similarly as in the case of integral-dimensional (nonrandom) measures (see, e.g., Preiss [13]) $P$ may be interpreted as the distribution of the random tangential measure of $\varphi$ at the typical point of the Cantor set. (Of course, the notion "tangential" becomes more appropriate for higher-dimensional measures.)

\section{The Palm Property of the tangential Measure}

This section will not be needed for the rest of the paper. The reader who is interested only in the density of the Cantor measure may omit it. For all others we give here a further interpretation of the distribution of the random tangential measure. In Ulrich Zähle [17] the following invariance property of $P$ with respect to the translations $\left(T_{x}\right)_{x \in \mathbf{R}}$ is shown:

$$
\iint f\left(T_{x} \mu,-x\right) \mu(d x) P(d \mu)=\iint f(\mu, x) \mu(d x) P(d \mu) .
$$

(By the scaling property it suffices to prove the equation for $P_{0}$ instead of $P$ for which it follows from its definition.) This invariance characterizes $P$ as the Palm distribution of a uniquely determined $\left(T_{x}\right)$-invariant $\sigma$-finite measure $Q$ on the space of locally finite measures on $\mathbf{R}$ with finite intensity $\lambda=\int([0,1]) Q(d \mu)$ (cf. Mecke [10]). This means that

$$
P=\lambda^{-1} \iint_{0}^{1} 1_{(\cdot)}\left(T_{x} \mu\right) \mu(d x) Q(d \mu) .
$$

(Note that in our case the "quasi-distribution" $Q$ is not finite. For more details see the paper [17] mentioned above.) Hence, $P$ may be interpreted as the distribution arising from $Q$ by translation of the origin into a point chosen "at random" according to the measure underlying the quasi-distribution $Q$, i.e., the origin may be considered as the typical point of the support of the random tangential measure of $\varphi$ with distribution $P$. (A good introduction into the theory of Palm distributions may be found in Stoyan, Kendall, and Mecke [14].) In general, such measures like $P$, i.e., Palm distributions satisfying the scaling property (4) for some $D$ with respect to the origin are called in Ulrich Zähle [17] $D$-scale invariant, since by the above arguments the origin there is the typical 
point of observation. (This is an axiomatic approach to $D$-scale invariance as used in physics.)

\section{THE FRACTIONAL DENSITY OF THE CANTOR MEASURE}

The concept of (upper and lower) densities of geometric measures plays an important role in the corresponding theory. Its connection with rectifiability in the integral-dimensional case has been a large field of research in geometric measure theory (for references, see Federer [4], Preiss [13]). Therefrom we know that in the fractional case the usual densities do not exist. Bedford and Fisher [1] first introduced the concept of Cesáro densities (called there secondorder densities) on the example of Cantor-type measures and of the measure of the zeros of Brownian motion on $[0,1]$. We will give here another proof of existence of the right and left densities of the middle-third Cantor measure

$$
\begin{aligned}
& d_{D}^{+} \varphi(x):=\lim _{T \rightarrow \infty} \frac{1}{T} \int_{0}^{T} \frac{\varphi\left(\left[x, x+e^{-t}\right]\right)}{e^{-t D}} d t, \\
& d_{D}^{-} \varphi(x):=\lim _{T \rightarrow \infty} \frac{1}{T} \int_{0}^{T} \frac{\varphi\left(\left[x-e^{-t}, x\right]\right)}{e^{-t D}} d t
\end{aligned}
$$

at $\varphi$-a.a. $x \in[0,1]$. We write

$$
d_{D} \varphi(x):=2^{-D}\left(d_{D}^{+} \varphi(x)+d_{D}^{-} \varphi(x)\right)
$$

for the symmetric density. Note that these densities may also be considered as the $D$-fractional Cesáro derivatives of the Cantor function $\Phi(x)$ at almost all $x$ from the Cantor set.

Theorem 1. At $\varphi$-a.a. $x \in[0,1]$ we have

$$
d_{D}^{+} \varphi(x)=d_{D}^{-} \varphi(x)=\int_{0}^{1} \int 2^{r} \varphi\left(\left[y, y+3^{-r}\right]\right) \varphi(d y) d r .
$$

Proof of Theorem 1. For any locally finite measure $\mu$ on $\mathbf{R}$ we have

$$
\begin{aligned}
\lim _{T \rightarrow \infty} & \frac{1}{T} \int_{0}^{T} \frac{\mu\left(\left[0, e^{-t}\right]\right)}{e^{-t D}} d t=\lim _{\substack{N \rightarrow \infty \\
(N \in \mathbf{N})}} \frac{1}{N} \int_{0}^{N} \frac{\mu\left(\left[0, e^{-t}\right]\right)}{e^{-t D}} d t \\
= & \lim _{\substack{N \rightarrow \infty \\
(N \in \mathbb{N})}} \frac{1}{N} \int_{0}^{N} \frac{\mu\left(\left[0,3^{-t}\right]\right)}{3^{-t D}} d t
\end{aligned}
$$

provided that the limit exists. The right-hand side equals

$$
\begin{aligned}
\lim _{N \rightarrow \infty} & \frac{1}{N} \sum_{n=0}^{N-1} \int_{0}^{1} 2^{r} 2^{n} \mu\left(3^{-n} 3^{-r}[0,1]\right) d r \\
= & \lim _{N \rightarrow \infty} \frac{1}{N} \sum_{n=0}^{N-1} \int_{0}^{1} 2^{r}\left(S_{D, 3}^{n} \mu\right)\left(3^{-r}[0,1]\right) d r .
\end{aligned}
$$

Since the distribution $P$ of the random tangential measure of $\varphi$ is $S_{D, 3^{-}}$ invariant, Birkhoff's ergodic theorem implies that the last limit exists for $P$-a.a. $\mu$ and its expectation equals

$$
\iint_{0}^{1} 2^{r} \mu\left(3^{-r}[0,1]\right) d r P(d \mu) .
$$


In view of (2) we may replace $P$ by $P_{0}$ and, therefore, the expection agrees with

$$
\int_{0}^{1} 2^{r} \int \varphi\left(\left[y, y+3^{-r}\right]\right) \varphi(d y) d r
$$

Below we will prove that $P$ is ergodic with respect to $S_{D, 3}$ when restricted to events depending only on the values of the measures on $[0,1]$. In this case we get

$$
\lim _{T \rightarrow \infty} \frac{1}{T} \int_{0}^{T} \frac{\mu\left(\left[0, e^{-t}\right]\right)}{e^{-t D}} d t=\int_{0}^{1} 2^{r} \int \varphi\left(\left[y, y+3^{-r}\right]\right) \varphi(d y) d r
$$

for $P_{0}$-a.a. $\mu$. Let $E$ be the set of all normed measures $\mu$ on $\mathbf{R}$ satisfying the last equation. Then we have

$$
0=P_{0}\left(E^{c}\right)=\int_{0}^{1} 1_{E^{c}}\left(T_{x} \varphi\right) \varphi(d x),
$$

i.e., $T_{x} \varphi \in E$ for $\varphi$-a.a. $x \in[0,1]$. Consequently, at these $x$ the right density $d_{D}^{+} \varphi(x)$ exists and takes the value in the assertion. Analogously, one can prove that

$$
d_{D}^{-} \varphi(x)=\int_{0}^{1} 2^{r} \int \varphi\left(\left[y-3^{-r}, y\right]\right) \varphi(d y) d r
$$

at $\varphi$-a.a. $x$, which coincides with the expression for $d_{D}^{+} \varphi(x)$ by symmetry.

It remains to show ergodicity. Define the function $g:\{0,1\}^{\mathbf{N}} \rightarrow[0,1]$ by

$$
g(\sigma)=\sum_{i=1} \frac{\sigma_{i} 2}{3^{i}} \text { if } \sigma=\left(\sigma_{1}, \sigma_{2}, \ldots\right) .
$$

Let $\left[\{0,1\}^{\mathbf{N}}, \nu, \boldsymbol{\theta}\right]$ be the Bernoulli system, i.e., $\nu$ is the product measure of the uniform distribution on $\{0,1\}$ and $\Theta$ is the shift

$$
\Theta\left(\sigma_{1}, \sigma_{2}, \ldots\right)=\left(\sigma_{2}, \sigma_{3}, \ldots\right) \text {. }
$$

From the construction in $\S 1$ we infer

$$
\varphi=\nu \circ g^{-1} \text {. }
$$

Let $A$ be an arbitrary measurable set on the space of locally finite measures on $\mathbf{R}$ depending only on the values of the measures on $[0,1]$. By the above arguments we get for any $x=g(\sigma) \in[0,1]$ and $\delta$-measure $\delta_{z}$

$$
\begin{aligned}
1_{S_{D, 3}^{-1} A}\left(T_{x} \varphi\right) & =1_{S_{D, 3}^{-1} A}\left(\int \delta_{\sum_{i=1}^{\infty}\left(\tau_{i}-\sigma_{i}\right) 2 / 3^{i}} \nu(d \tau)\right) \\
& =1_{A}\left(2 \int \delta_{\sum_{i=1}^{\infty}\left(\tau_{i}-\sigma_{i}\right) 2 / 3^{i-1}} \nu(d \tau)\right) \\
& =1_{A}\left(2 \int \delta_{\sum_{i=0}^{\infty}\left(\tau_{i+1}-\sigma_{i+1}\right) 2 / 3^{i}} L[0,1] \nu(d \tau)\right) \\
& =1_{A}\left(2 \int 1_{\left\{\sigma_{1}\right\}}\left(\tau_{1}\right) \delta_{\sum_{i=1}^{\infty}\left(\tau_{i+1}-\sigma_{i+1}\right) 2 / 3^{i}} \nu(d \tau)\right) \\
& =1_{A}\left(\int \delta_{\sum_{i=1}^{\infty}\left(\tau_{i+1}-\sigma_{i+1}\right) 2 / 3^{i}} \nu(d \tau)\right) \\
& =1_{A}\left(\int \delta_{\sum_{i=1}^{\infty}\left(\tau_{i}-\sigma_{i+1}\right) 2 / 3^{i}} \nu(d \tau)\right) .
\end{aligned}
$$


Similarly,

Denote

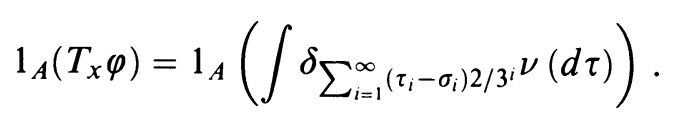

$$
\Xi:=\left\{\sigma \in\{0,1\}^{\mathbf{N}}: \int \delta_{\sum_{i=1}^{\infty}\left(\tau_{i}-\sigma_{i}\right) 2 / 3^{i}} \nu(d \tau) \in A\right\} .
$$

Then we have by the definition of $P_{0}$ that

$$
P(A)=P_{0}(A)=\nu(\Xi) .
$$

Suppose additionally that $A$ is a $P$-almost $S_{D, 3}$-invariant set, i.e.,

$$
P\left(S_{D, 3}^{-1} A \Delta A\right)\left(=P_{0}\left(S_{D, 3}^{-1} A \Delta A\right)\right)=0 .
$$

In this case the above equations yield

$$
\nu\left(\Theta^{-1} \Xi \Delta \Xi\right)=P_{0}\left(S_{D, 3}^{-1} A \Delta A\right)=0 .
$$

Because of ergodicity of the Bernoulli flow, it follows that $\nu(\Xi)=0$ or 1 . Hence, $P(A)=0$ or 1 .

\section{COMPUTATION OF THE DENSITY}

Our final aim is to find the numerical value of $d_{D} \varphi$. We first transform the integral in Theorem 1 as follows:

$$
\begin{aligned}
\int_{0}^{1} 2^{r} \int \varphi\left(\left[y, y+3^{-r}\right]\right) d r \varphi(d y) \\
=\int_{0}^{1} 2^{r} \iint 1_{[0,1 / 3]}(x-y) \varphi(d x) \varphi(d y) d r \\
\quad+\int_{0}^{1} 2^{r} \iint 1_{\left[1 / 3,3^{-r}\right]}(x-y) \varphi(d x) \varphi(d y) d r \\
=\frac{1}{\ln 2} \iint 1_{[0,1 / 3]}(x-y) \varphi(d x) \varphi(d y) \\
\quad+\iint 1_{[1 / 3,1]}(x-y) \int 1_{[0,-\ln (x-y) / \ln 3]}(r) 2^{r} d r \varphi(d x) \varphi(d y) \\
=\frac{1}{\ln 2} \frac{1}{4}+\frac{1}{\ln 2} \iint 1_{[1 / 3,1]}(x-y)\left((x-y)^{-D}-1\right) \varphi(d x) \varphi(d y) \\
=\frac{1}{\ln 2} \frac{1}{4}+\frac{1}{\ln 2} \int_{0}^{1 / 3} \int_{2 / 3}^{1}(x-y)^{-D} \varphi(d x) \varphi(d y)-\frac{1}{\ln 2} \frac{1}{4} \\
=\frac{1}{\ln 2} \int_{0}^{1 / 3} \int_{2 / 3}^{1}(x-y)^{-D} \varphi(d x) \varphi(d y) .
\end{aligned}
$$

Because of (1) and symmetry, the last expression equals

$$
\frac{1}{2 \ln 2} \iint(u+v+1)^{-D} \varphi(d u) \varphi(d v) .
$$

By use of (6) we may continue with

$$
\frac{1}{2 \ln 2} \iint\left(\sum_{i=1}^{\infty}\left(\sigma_{i}+\tau_{i}\right) \cdot \frac{2}{3^{i}}+1\right)^{-D} \nu(d \sigma) \nu(d \tau) .
$$


Defining i.i.d. random variables $\xi_{1}, \xi_{2}, \ldots$ with $\mathbf{P}\left\{\xi_{i}=0\right\}=\frac{1}{2}, \mathbf{P}\left\{\xi_{i}=-1\right\}=$ $\mathbf{P}\left\{\xi_{i}=1\right\}=\frac{1}{4}$ and putting

$$
\eta:=\sum_{i=1}^{\infty} \xi_{i} \cdot \frac{2}{3^{i}}
$$

we obtain

$$
d_{D}^{+} \varphi=(2 \ln 2)^{-1} \mathbf{E}(\eta+2)^{-D} .
$$

Since the function $(x+2)^{-D}$ for $|x|<2$ is representable by

$$
(x+2)^{-D}=2^{-D}\left(1+\sum_{n=1}^{\infty}(-1)^{n} \frac{D(D+1) \cdots(D+n-1)}{n !} \cdot \frac{1}{2^{n}} \cdot x^{n}\right)
$$

and $|\eta|<1$, it remains to calculate the moments of the random variable $\eta$. They are equal to

$$
\mathbf{E} \eta^{2 k-1}=0
$$

(7) $\quad \mathbf{E} \eta^{2 k}=\sum_{l=1}^{k} \sum_{\substack{i_{1}+\cdots+i_{l}=k, i_{j} \geq 1}} 2^{2 k-1} \frac{(2 k) !}{\left(2 i_{1}\right) ! \cdots\left(2 i_{l}\right) !}$

$$
\times\left[\left(3^{2 i_{1}}-1\right)\left(3^{2 i_{1}+2 i_{2}}-1\right) \cdots\left(3^{2 i_{1}+\cdots+2 i_{l}}-1\right)\right]^{-1} .
$$

Thus, we have proved

\section{Theorem 2.}

$$
d_{D}^{-} \varphi=d_{D}^{+} \varphi=\frac{1}{2 \ln 2} \frac{1}{2^{D}}\left(1+\sum_{k=1}^{\infty} \frac{D(D+1) \cdots(D+2 k-1)}{(2 k) !} \cdot \frac{1}{4^{k}} \cdot \mathbf{E} \eta^{2 k}\right)
$$

where $\mathbf{E} \eta^{2 k}$ is given by (7). The corresponding numerical values of the densities are $d_{D}^{+} \varphi=0.48272 \ldots$ and $d_{D} \varphi=0.62344 \ldots$.

\section{REFERENCES}

1. T. Bedford and A. Fisher, Analogues of the Lebesgue density theorem for fractal sets of reals and integers, Preprint, Delft Univ. of Tech., Report of the Dept. of Tech. Math. and Infor. No. 89-88, 1989.

2. T. Bedford and T. Kamae, Stieltjes integration and stochastic calculus with respect to selfaffine functions, Preprint, Delft Univ. of Tech., Report of the Faculty of Tech. Math. and Infor. No. 90-24, 1990.

3. K. Falconer, Fractal geometry, Wiley, 1990.

4. H. Federer, Colloquium lectures on geometric measure theory, Bull. Amer. Math. Soc. 84 (1978), 291-338.

5. T.-Y. Hu and K.-S. Lau, The sum of Rademacher functions and Hausdorff dimension, Math. Proc. Cambridge Philos. Soc. (to appear).

6. Fractal dimension and singularities of the Weierstrass type functions, preprint.

7. T. Kamae, A characterization of self-affine functions, Japan J. Appl. Math. 3 (1986), 271280.

8. N. Kôno, On self-affine functions, Japan J. Appl. Math. 3 (1986), 259-269. 
9. __ On self-affine functions. II, Japan J. Appl. Math. 5 (1988), 441-454.

10. J. Mecke, Stationäre zufällige Maße auf lokalkompakten abelschen Gruppen, Z. Wahrsch. Verw. Gebiete 9 (1967), 36-58.

11. N. Patzschke and M. Zähle, Fractional differentiation in the self-affine case I. Random functions, Stochastic Process Appl. (to appear).

12. N. Pazschke and U. Zähle, Self-similar random measures IV. The recursive construction model of Falconer, Graf, and Mauldin and Williams, Math. Nachr. 149 (1990), 285-302.

13. D. Preiss, Geometry of measures in $\mathbf{R}^{n}$ : Distribution, rectifiability, and densities, Ann. of Math. (2) 125 (1987), 537-643.

14. D. Stoyan, W. S. Kendall, and J. Mecke, Stochastic geometry and its applications, Wiley, 1987.

15. C. Tricot, Rectifiable and fractal sets, CRM-1626, preprint.

16. __ Local convex envelopes of a curve, and the value of its fractal dimension, preprint.

17. U. Zähle, Self-similar random measures I. Notion, carrying Hausdorff dimension and hyperbolic distribution, Probab. Theory Related Fields 80 (1988), 79-100.

Faculty of Mathematics, University of Jena, 0-6900 Jena, Germany 